Kocaeli Journal of Science and Engineering

\title{
Investigation of Stability Changes in a Neural Field Model
}

\author{
Berrak ÖZGÜR ${ }^{1, *}$ \\ ${ }^{1}$ İzmir Democracy University, Department of Mathematics, Izmir, 35140, Turkey, ORCID: 0000-0002-9709-7376
}

Article Info

Research paper
Received $\quad:$ January 04,2021
Accepted $\quad:$ February 08,2021

\begin{abstract}
In this paper, the stability analysis of the neural field model is studied. The special case for three neuron populations is considered. The work is conducted by finding the characteristic equation of the system first and then investigating the characteristic roots of the third-order equation by using the Routh-Hurwitz criterion and Sturm sequence. The main analysis is given in two parts considering the nonexistence and existence of the delay term. Some basic stability criteria in terms of coefficients of the system are given in the theorems.
\end{abstract}

Keywords
Characteristic Equation
Neural Field Model
Routh-Hurwitz Criterion
Stability Analysis
Sturm Sequence

\section{Introduction}

The term 'dynamical system' is used to determine a system varying with respect to time. In applied mathematics, in order to understand the general construction for a real-world phenomenon and analyse its future state, mathematical models are used. Differential equations, difference equations and functional equations are frequently used when writing mathematical models for dynamical systems representing the real phenomena. Hence, the important analyses related to them can be made by using convenient mathematical methods.

In sciences such as biology, engineering, economics, since time is very important, we generally use a time delay in writing more realistic models. The theory of delay differential equations has an important role in such fields.

The scientists aimed to model the activity of large neuron populations in the brain, use the neural field models. These models are constructed using integro-

\footnotetext{
${ }^{*}$ Corresponding Author: berrak.ozgur@idu.edu.tr
}

differential equations including a time delay. For the basic facts in neural field models, one can refer to the studies given in $[1,2]$. Besides these studies, the stability analysis of the neural field model and the existence and uniqueness of their solutions are studied in some papers [3-15].

In stability analysis, obtaining the characteristic equation of the system and determining the characteristic roots construct the important part. There are some studies on investigation of the stability analysis for this model including functional analysis and numerical methods $[7,9,10]$. In [11-15] the analysis is made by using the Dcurves method and the Routh-Hurwitz criterion.

In this study, we are interested in the stability of a neural field model for three neuron populations. The general overview of the study is given in the following; the model is given in Section 2. The stability properties for the model are given in Section 3. The roles of the system parameters on the stability of the model are shown. This analysis is made by using the Sturm sequence since the corresponding characteristic equation of the model is third order. The conclusion of this study is in Section 4. 


\section{Neural Field Model}

The scientists use neural field equations to model dynamics of mean membrane potential for $p$ neural populations on the space $\Omega \subset R^{d}$. This model given in $[6,7,9,10]$ is given below

$\left(\frac{d}{d t}+l_{i}\right) V_{i}(t, r)=\sum_{j=1}^{p} \int_{\Omega} J_{i j}(r, \bar{r}) S\left[\sigma_{j}\left(V_{j}(t-\right.\right.$ $\left.\left.\left.\tau_{i j}(r, \bar{r}), \bar{r}\right)-h_{j}\right)\right] d \bar{r}+I_{i}^{e x t}(r, t), t \geq 0,1 \leq i \leq p$

$V_{i}(t, r)=\phi_{i}(t, r), t \in[-T, 0]$

Here, we consider this model for three neuron populations $(p=3)$. The synaptic inputs for large groups of neurons at position $x$ and time $t$ are represented by the functions $V_{1}(x, t), V_{2}(x, t)$ and $V_{3}(x, t)$. We consider $\Omega=$ $\left[-\frac{\pi}{2}, \frac{\pi}{2}\right]$. The relations of neurons on different populations are shown by the functions $J_{i j}(x, y)$. Some of the relations among them are restricted. The stability analysis is made in the special case that $J_{12}(x, y) \neq 0, J_{21}(x, y) \neq 0$, $J_{23}(x, y) \neq 0, J_{32}(x, y) \neq 0$. In this research, the delay term is considered constant as $\tau(x, y)=\tau$. Hence the linearized model near $(0,0,0)$ is given below. For this model the functions $U_{1}(x, t), U_{2}(x, t)$ and $U_{3}(x, t)$ are used.

$\frac{d}{d t} U_{1}(x, t)+l_{1} U_{1}(x, t)=\sigma_{2} s_{1} \int_{-\frac{\pi}{2}}^{\frac{\pi}{2}} J_{12}(x, y) U_{2}(y, t-$ $\tau(x, y)) d y$

$\frac{d}{d t} U_{2}(x, t)+l_{2} U_{2}(x, t)=\sigma_{1} s_{1} \int_{-\frac{\pi}{2}}^{\frac{\pi}{2}} J_{21}(x, y) U_{1}(y, t-$ $\tau(x, y)) d y+\sigma_{3} s_{1} \int_{-\frac{\pi}{2}}^{\frac{\pi}{2}} J_{23}(x, y) U_{3}(y, t-\tau(x, y)) d y$

$\frac{d}{d t} U_{3}(x, t)+l_{3} U_{3}(x, t)=\sigma_{2} s_{1} \int_{-\frac{\pi}{2}}^{\frac{\pi}{2}} J_{32}(x, y) U_{2}(y, t-$

$\tau(x, y)) d y$

\section{Stability Analysis}

Considering the Fourier method, we are looking for the solutions as $U_{1}(x, t)=e^{i k x} u_{1}(t), \quad U_{2}(x, t)=$ $e^{i k x} u_{2}(t), \quad U_{3}(x, t)=e^{i k x} u_{3}(t)$. Here $u_{1}(t)=c_{1} e^{\lambda t}$, $u_{2}(t)=c_{2} e^{\lambda t}$ and $u_{3}(t)=c_{3} e^{\lambda t}$. Writing them in the system (2), we get the following system

$\lambda e^{i k x} u_{1}(t)+l_{1} e^{i k x} u_{1}(t)=$ $\sigma_{2} s_{1} e^{-\lambda \tau} u_{2}(t) \int_{-\frac{\pi}{2}}^{\frac{\pi}{2}} J_{12}(x, y) e^{i k y} d y$

$$
\begin{aligned}
& \lambda e^{i k x} u_{2}(t)+l_{2} e^{i k x} u_{2}(t)= \\
& \sigma_{1} s_{1} e^{-\lambda \tau} u_{1}(t) \int_{-\frac{\pi}{2}}^{\frac{\pi}{2}} J_{21}(x, y) e^{i k y} d y+ \\
& \sigma_{3} s_{1} e^{-\lambda \tau} u_{3}(t) \int_{-\frac{\pi}{2}}^{\frac{\pi}{2}} J_{23}(x, y) e^{i k y} d y \\
& \lambda e^{i k x} u_{3}(t)+l_{3} e^{i k x} u_{3}(t)= \\
& \sigma_{2} s_{1} e^{-\lambda \tau} u_{2}(t) \int_{-\frac{\pi}{2}}^{\frac{\pi}{2}} J_{32}(x, y) e^{i k y} d y
\end{aligned}
$$

The solutions of the system are the functions $\cos (2 n x)$ and $\sin (2 n x)$ [9]. Hence we have

$$
\begin{aligned}
& \lambda u_{1}(t)+l_{1} u_{1}(t)=\sigma_{2} s_{1} e^{-\lambda \tau} u_{2}(t) \int_{-\frac{\pi}{2}}^{\frac{\pi}{2}} J_{12}(x, y) e^{i k y} d y \\
& \lambda u_{2}(t)+l_{2} u_{2}(t)=\sigma_{1} s_{1} e^{-\lambda \tau} u_{1}(t) \int_{-\frac{\pi}{2}}^{\frac{\pi}{2}} J_{21}(x, y) e^{i k y} d y+ \\
& \sigma_{3} s_{1} e^{-\lambda \tau} u_{3}(t) \int_{-\frac{\pi}{2}}^{\frac{\pi}{2}} J_{23}(x, y) e^{i k y} d y \\
& \lambda u_{3}(t)+l_{3} u_{3}(t)=\sigma_{2} s_{1} e^{-\lambda \tau} u_{2}(t) \int_{-\frac{\pi}{2}}^{\frac{\pi}{2}} J_{32}(x, y) e^{i k y} d y
\end{aligned}
$$

Considering the coefficient determinant of this system with respect to $u_{1}(t), u_{2}(t)$ and $u_{3}(t)$, we get the following characteristic equation arranged in terms of the powers of $\lambda$

$$
\begin{aligned}
& \lambda^{3}+\left(l_{1}+l_{2}+l_{3}\right) \lambda^{2}+\left(l_{2} l_{3}+l_{1} l_{3}+l_{1} l_{2}\right) \lambda+ \\
& l_{1} l_{2} l_{3}-K_{1} K_{3} F_{3} F_{4} e^{-2 \lambda \tau}\left(\lambda+l_{1}\right)-K_{1} K_{2} F_{1} F_{2} e^{-2 \lambda \tau}(\lambda+ \\
& \left.l_{3}\right)=0
\end{aligned}
$$

where $K_{1}=\sigma_{2} s_{1}, K_{2}=\sigma_{1} s_{1}, K_{3}=\sigma_{3} s_{1}$,

$$
\begin{aligned}
& F_{1}=\int_{-\frac{\pi}{2}}^{\frac{\pi}{2}} J_{12}(x, y) e^{i k y} d y, \\
& F_{2}=\int_{-\frac{\pi}{2}}^{\frac{\pi}{2}} J_{21}(x, y) e^{i k y} d y, \\
& F_{3}=\int_{-\frac{\pi}{2}}^{\frac{\pi}{2}} J_{23}(x, y) e^{i k y} d y, \\
& F_{4}=\int_{-\frac{\pi}{2}}^{\frac{\pi}{2}} J_{32}(x, y) e^{i k y} d y
\end{aligned}
$$

If there is no delay term in the system, i.e., $\tau=0$, the characteristic equation turns into the following form 
$\lambda^{3}+\left(l_{1}+l_{2}+l_{3}\right) \lambda^{2}+\left(l_{2} l_{3}+l_{1} l_{3}+l_{1} l_{2}-K_{1} K_{3} F_{3} F_{4}-\right.$ $\left.K_{1} K_{2} F_{1} F_{2}\right) \lambda+l_{1} l_{2} l_{3}-K_{1} K_{3} F_{3} F_{4} l_{1}-K_{1} K_{2} F_{1} F_{2} l_{3}=0$

According to the Routh-Hurwitz criterion, we may give the following theorem.

Theorem: Consider the system (2). If the following conditions are satisfied

$l_{1}+l_{2}+l_{3}>0$,

$l_{1} l_{2} l_{3}-K_{1} K_{3} F_{3} F_{4} l_{1}-K_{1} K_{2} F_{1} F_{2} l_{3}>0$

and

$\left(l_{1}+l_{2}+l_{3}\right)\left(l_{2} l_{3}+l_{1} l_{3}+l_{1} l_{2}-K_{1} K_{3} F_{3} F_{4}-\right.$

$\left.K_{1} K_{2} F_{1} F_{2}\right)-\left(l_{1} l_{2} l_{3}-K_{1} K_{3} F_{3} F_{4} l_{1}-K_{1} K_{2} F_{1} F_{2} l_{3}\right)>0$

then the system is stable near $(0,0,0)$ in the absence of delay term.

Proof: If the conditions given above are satisfied then, according to the Routh-Hurwitz criterion, all roots of the characteristic equation have negative real parts and the system is stable near $(0,0,0)$ in the absence of delay term.

In case of existence of a delay term, we apply the procedure given in [16]. Because of the critical delays, some characteristic roots change from having negative real parts to having positive real parts. For this reason, we will examine the purely imaginary roots $\lambda=i \sigma$. To get the characteristic equation and see such a change, we substitute $\lambda=i \sigma$ in Eq. (5), and separating the real and imaginary parts, we get

$-\sigma^{2}\left(l_{1}+l_{2}+l_{3}\right)+l_{1} l_{2} l_{3}-K_{1} K_{3} F_{3} F_{4} \sigma \sin (2 \sigma \tau)-$ $K_{1} K_{3} F_{3} F_{4} l_{1} \cos (2 \sigma \tau)-K_{1} K_{2} F_{1} F_{2} \sigma \sin (2 \sigma \tau)-$ $K_{1} K_{2} F_{1} F_{2} l_{3} \cos (2 \sigma \tau)=0$

$-\sigma^{3}+\sigma\left(l_{2} l_{3}+l_{1} l_{3}+l_{1} l_{2}\right)-K_{1} K_{3} F_{3} F_{4} \sigma \cos (2 \sigma \tau)+$ $K_{1} K_{3} F_{3} F_{4} l_{1} \sin (2 \sigma \tau)-K_{1} K_{2} F_{1} F_{2} \sigma \cos (2 \sigma \tau)+$ $K_{1} K_{2} F_{1} F_{2} l_{3} \sin (2 \sigma \tau)=0$

Rearranging them we get the following two equations

$$
\begin{aligned}
& -\sigma^{2}\left(l_{1}+l_{2}+l_{3}\right)+l_{1} l_{2} l_{3}=K_{1} K_{3} F_{3} F_{4} \sigma \sin (2 \sigma \tau)+ \\
& K_{1} K_{3} F_{3} F_{4} l_{1} \cos (2 \sigma \tau)+K_{1} K_{2} F_{1} F_{2} \sigma \sin (2 \sigma \tau)+ \\
& K_{1} K_{2} F_{1} F_{2} l_{3} \cos (2 \sigma \tau)
\end{aligned}
$$

$-\sigma^{3}+\sigma\left(l_{2} l_{3}+l_{1} l_{3}+l_{1} l_{2}\right)=K_{1} K_{3} F_{3} F_{4} \sigma \cos (2 \sigma \tau)-$ $K_{1} K_{3} F_{3} F_{4} l_{1} \sin (2 \sigma \tau)+K_{1} K_{2} F_{1} F_{2} \sigma \cos (2 \sigma \tau)-$ $K_{1} K_{2} F_{1} F_{2} l_{3} \sin (2 \sigma \tau)$

Taking squares of both sides in these two equations and adding them we get the polynomial equation given below

$\sigma^{6}+\left(l_{1}^{2}+l_{2}{ }^{2}+l_{3}^{2}\right) \sigma^{4}+\left(l_{2}^{2} l_{3}{ }^{2}+l_{1}^{2}{l_{3}}^{2}+l_{1}{ }^{2} l_{2}{ }^{2}-\right.$ $K_{1}{ }^{2} K_{3}{ }^{2}{F_{3}}_{3}{ }^{2} F_{4}{ }^{2}-2 K_{1}{ }^{2} K_{2} K_{3} F_{1} F_{2} F_{3} F_{4}-$ $\left.K_{1}{ }^{2} K_{2}{ }^{2}{F_{1}}^{2}{F_{2}}^{2}\right) \sigma^{2}+l_{1}{ }^{2} l_{2}{ }^{2} l_{3}{ }^{2}-K_{1}{ }^{2} K_{3}{ }^{2}{F_{3}}^{2}{F_{4}}^{2} l_{1}{ }^{2}-$ $2 K_{1}^{2} K_{2} K_{3} F_{1} F_{2} F_{3} F_{4} l_{1} l_{3}-K_{1}{ }^{2} K_{2}{ }^{2} F_{1}{ }^{2} F_{2}{ }^{2} l_{3}{ }^{2}=0$

Now following the Routh-Hurwitz criterion, we replace $\mu$ by $\sigma^{2}$. Hence we have the following third-order polynomial equation to carry the stability analysis for the model.

$$
\mu^{3}+\left(l_{1}^{2}+l_{2}^{2}+l_{3}^{2}\right) \mu^{2}+\left(l_{2}^{2} l_{3}^{2}+l_{1}^{2} l_{3}^{2}+\right.
$$
$l_{1}^{2} l_{2}^{2}-K_{1}^{2} K_{3}{ }^{2} F_{3}{ }^{2} F_{4}{ }^{2}-2 K_{1}{ }^{2} K_{2} K_{3} F_{1} F_{2} F_{3} F_{4}-$ $\left.K_{1}{ }^{2} K_{2}{ }^{2}{F_{1}}^{2} F_{2}{ }^{2}\right) \mu+l_{1}{ }^{2} l_{2}{ }^{2} l_{3}{ }^{2}-K_{1}{ }^{2} K_{3}{ }^{2} F_{3}{ }^{2} F_{4}{ }^{2} l_{1}{ }^{2}-$ $2 K_{1}{ }^{2} K_{2} K_{3} F_{1} F_{2} F_{3} F_{4} l_{1} l_{3}-K_{1}{ }^{2} K_{2}{ }^{2} F_{1}{ }^{2}{F_{2}}_{2}{ }^{2} l_{3}{ }^{2}=0$

For simplicity, we call the coefficients

$$
\begin{aligned}
& A=l_{1}{ }^{2}+l_{2}{ }^{2}+l_{3}{ }^{2} \\
& B=l_{2}{ }^{2} l_{3}{ }^{2}+l_{1}{ }^{2} l_{3}{ }^{2}+l_{1}{ }^{2}{l_{2}}^{2}-K_{1}{ }^{2} K_{3}{ }^{2}{F_{3}}_{3}{ }^{2}{ }_{4}{ }^{2}- \\
& 2 K_{1}{ }^{2} K_{2} K_{3} F_{1} F_{2} F_{3} F_{4}-K_{1}{ }^{2} K_{2}{ }^{2}{F_{1}}^{2}{F_{2}}^{2} \\
& C=l_{1}{ }^{2} l_{2}{ }^{2} l_{3}{ }^{2}-K_{1}{ }^{2}{K_{3}}^{2}{F_{3}}^{2}{F_{4}}^{2} l_{1}{ }^{2}- \\
& 2 K_{1}{ }^{2} K_{2} K_{3} F_{1} F_{2} F_{3} F_{4} l_{1} l_{3}-K_{1}{ }^{2} K_{2}{ }^{2}{F_{1}}^{2}{F_{2}}^{2} l_{3}{ }^{2}
\end{aligned}
$$

Since the leading coefficient is positive, a positive real root may occur in two cases.

i) If $C<0$ then the positive real root occurs.

ii) If $C>0$ then a negative real root is guaranteed. To analyze the possibility to have two positive real roots, we use the Sturm sequence of the polynomial in Eq. (12).

The details of the method of Sturm sequence are constructed by determining whether a positive real root exists. After finding the functions in the Sturm sequence, the sign changes in endpoints of the considered interval must be determined. The number gives us the number of 
real roots. After this step, the conditions must be analyzed to see the positive real root.

We will follow the procedure given in [16]. Let us start with the polynomials

$f_{0}=\mu^{3}+A \mu^{2}+B \mu+C$

and

$f_{1}=3 \mu^{2}+2 A \mu+B$

where $f_{1}=f_{0}{ }^{\prime}$. Applying the division algorithm

$f_{0}=q_{0} f_{1}+f_{2}$

$f_{1}=q_{1} f_{2}+f_{3}$

we have

$f_{2}=\left(\frac{2}{9} A^{2}-\frac{2}{3} B\right) \mu+C-\frac{1}{9} A B$

$f_{3}=-\frac{9}{4} \frac{4 B^{3}-A^{2} B^{2}-18 A B C+4 C A^{3}+27 C^{2}}{\left(A^{2}-3 B\right)^{2}}$

By considering the sign changes at each endpoint of the interval $(-\infty, \infty)$, we may construct the following table given in [16] to have three sign changes, hence three real roots for the case (ii).

Table 1. The sign changes for the Sturm sequence.

\begin{tabular}{ccc}
\hline & $-\infty$ & $\infty$ \\
\hline$f_{0}$ & - & + \\
\hline$f_{1}$ & + & + \\
\hline$f_{2}$ & - & + \\
\hline$f_{3}$ & + & +
\end{tabular}

In order to get this table we need the following conditions,

$A^{2}-3 B>0$

and

$4\left(B^{2}-3 A C\right)\left(A^{2}-3 B\right)-(9 C-A B)^{2}>0$ where the constants $A, B, C$ are determined as in (13).

And for the case (ii), there exists one positive real root if $A<0$ or $A>0$ and $B<0$ [16].

We will conclude this part by the following theorem based on the theorem in [16] in case of a delay term exists.

Theorem: Consider the characteristic equation (5) for the system (2) with a delay term. The system is unstable near $(0,0,0)$ if and only if $A, B$ and $C$ are not all positive and either $C<0$, or $C>0, A^{2}-3 B>0$ and $4\left(B^{2}-\right.$ $3 A C)\left(A^{2}-3 B\right)-(9 C-A B)^{2}>0$ is satisfied where $A, B$ and $C$ are given in (13).

Proof: In the existence of the conditions given above, we have three real characteristic roots and one of them is positive. Hence the system becomes unstable.

\section{Conclusion}

In this study, the stability properties of a neural field model are constructed in a special case. The linearized model for three neuron populations is considered and is investigated for the stability in an algebraic way. The main idea here is to determine the roots of the characteristic equation. Since the characteristic equation is third-order, the Routh-Hurwitz criterion and the Sturm sequence are used. These two methods give the chance to make the analysis in an efficient way. As shown in this study, the stability properties in terms of the coefficients on the system are determined in a quick way by two theorems.

\section{Conflict of Interests}

No conflict of interest was stated by the authors.

\section{Acknowledgements}

The author would like to thank the editor and the reviewers for their valuable suggestions and comments.

\section{Declaration of Ethical Standards}

The authors of this article declares that the materials and methods used in this study do not require ethical committee permission and legal-special permission.

\section{References}

[1] Wilson H., Cowan J., 1973. A Mathematical Theory of the Functional Dynamics of Cortical and Thalamic Nervous Tissue. Biological Cybernetics, 13(2), pp. 55-80. 
[2] Amari S.I., 1977. Dynamics of Pattern Formation in Lateral-inhibition Type Neural Fields. Biological Cybernetics, 27(2), pp. 77-87.

[3] Coombes S., 2005. Waves, Bumps, and Patterns in Neural Field Theories. Biological Cybernetics, 93(2), pp. 91-108.

[4] Atay F.M., Hutt A., 2006. Stability and Bifurcations in Neural Fields with Finite Propagation Speed and General Connectivity. Siam Journal on Mathematical Analysis, 5(4), pp. 670-698.

[5] Coombes S., Venkov N.A., Shiau L., Bojak L., Liley D.T.J., Laing C.R., 2007. Modeling Electrocortical Activity Through Improved Local Approximations of Integral Neural Field Equations. Physical Review E, 76, 051901 .

[6] Faye G., Faugeras O., 2010. Some Theoretical and Numerical Results for Delayed Neural Field Equations. Physica D: Nonlinear Phenomena, 239(9), pp. 561-578.

[7] Veltz R., Faugeras O., 2011. Stability of the Stationary Solutions of Neural Field Equations with Propagation Delay. Journal of Mathematical Neuroscience, 1, 1, pp. 1-28.

[8] Van Gils S.A., Janssens S.G., Kuznetsov Yu. A., Visser S., 2013. On Local Bifurcations in Neural Field Models with Transmission Delays. Journal of Mathematical Biology, 66(4), pp. 837-887.

[9] Veltz R., 2013. Interplay Between Synaptic Delays and Propagation Delays in Neural Field Equations.
Siam Journal of Applied Dynamical Systems, 12(3), pp. 1566-1612.

[10] Veltz R. Faugeras O., 2013. A Center Manifold Result for Delayed Neural Fields Equations. Siam Journal on Mathematical Analysis, 45(3), pp. 15271562 .

[11] Özgür B., Demir A., 2016. Some Stability Charts of a Neural Field Model of Two Neural Populations. Communications in Mathematics and Applications, 7(2), pp. 159-166.

[12] Özgür B., Demir A., Erman S., 2018. A Note on the Stability of a Neural Field Model. Hacettepe Journal of Mathematics and Statistics, 47(6), pp. 1495-1502.

[13] Özgür B., Demir A., 2018. On the Stability of Two Neuron Populations Interacting with Each Other. Rocky Mountain Journal of Mathematics, 48(7), pp. 2337-2346.

[14] Özgür B., 2019. Some stability notes of a neural field model. IDES 2019 International Design and Engineering Symposium, İzmir, Turkey, 10-12 October, pp. 123-126.

[15] Özgür B., 2020. Stability Switches in a Neural Field Model: An Algebraic Study on the Parameters. Sakarya University Journal of Science, 24(1), pp. 178-182.

[16] Forde J., Nelson P., 2004. Applications of Sturm Sequences to Bifurcation Analysis of Delay Differential Equation Models. Journal of Mathematical Analysis and Applications, 300, pp. 273-284. 